\title{
Posterior lens capsule abscess due to Propionibacterium acnes and Staphylococcus epidermidis following extracapsular cataract extraction
}

\author{
Ruth M Manners, Christopher R Canning
}

\begin{abstract}
A case of posterior lens capsular abscess occurring many months after an extracapsular cataract extraction is presented. This was caused by a mixed infection involving Propionibacterium acnes and Staphylococcus epidermidis. The significance of Staph epidermidis after such a long postoperative period is uncertain, but the case shows features typical of secondary endophthalmitis due to $P$ acnes, including a long delay in onset and a grumbling course not brought under control by medical treatment. It supports the theory that the nidus of infection is localised in the posterior lens capsule by showing development of a visible capsular abscess with associated vitreous involvement. The subsequent removal of the capsule and vitreous, despite leaving the intraocular lens in place, led to complete resolution of the inflammation. Both organisms have previously been found to be sequestered in the posterior lens capsule by histological and microbiological examination of excised capsular specimens. It is important to consider them as possible causative agents in the formation of a postoperative capsular abscess.
\end{abstract}

There is an increasing awareness that the differential diagnosis of chronic, indolent, postoperative endophthalmitis, especially following extracapsular cataract extraction, should include the anaerobe Propionibacterium acnes. This is a ubiquitous organism found on skin and conjunctiva ${ }^{1}$ and was generally previously dismissed as a contaminant. Propionibacteria are anaerobic, Gram-positive rods, and the organism may occur as a mixed infection as shown in the case presented here. It is highly sensitive to penicillin but less so to cephalosporins and resistant to gentamicin and vancomycin. ${ }^{2} P$ acnes has also been reported as causing intralenticular abscess ${ }^{3}$ and postoperative endophthalmitis following trabeculectomy and keratoplasty.

Staphylococcus epidermidis is an aerobic, Grampositive organism of low virulence, commonly found in normal eyes and causally associated with postoperative endophthalmitis since $1964 .^{4}$ Generally the delay between surgery and postoperative infection is a matter of days and is thought to be due to antibiotics and steroids quelling the initial response. Latent periods of several months are rare. ${ }^{5}$

We report here a case of delayed postcataract extraction endophthalmitis due to a mixed infection of $P$ acnes and Staph epidermidis. There are aspects of this case which suggest that the chronic infective source of the organisms in the eye was in the lens capsule.

In April 1989 a 79-year-old woman was admitted for left cataract extraction. Two years earlier a right extracapsular cataract extraction with posterior chamber lens implant had been performed, and her visual acuities then were $6 / 12$ in the right eye and hand movement in the left with appropriate myopic correction.

An uneventful left extracapsular cataract extraction and posterior chamber intraocular lens insertion was performed under local anaesthesia. The procedure included a corneal section, needle can-opener capsulotomy, nucleus expression by external pressure, irrigation and aspiration with the Simcoe cannula, and insertion of an American Medical Optics posterior chamber, 22 dioptre intraocular lens, model PC57B, under viscoelastic substance which was subsequently aspirated. The section was closed with five 10/0 monofilament nylon sutures and the eye padded. The patient was discharged on the second postoperative day on chloramphenicol eyedrops $0.5 \%$ and dexamethasone eyedrops $0 \cdot 1 \%$ four times a day.

The eye initially settled well following surgery, and five weeks postoperatively a visual acuity of 6/9 was achieved with appropriate correction. The eye appeared quiet and at this stage the posterior lens capsule was clear. All treatment was stopped.

Five months later the patient attended the casualty department complaining of pain and watering in the left eye for two days. On examination the left visual acuity was $6 / 12$ with glasses. Conjunctival injection was present, with a quiet anterior chamber. The posterior lens capsule was noted to be slightly thickened. A diagnosis of conjunctivitis was made and treatment with chloramphenicol eyedrops $0.5 \%$ four times a day was started again.

On review one week later the eye was still painful and a moderately severe anterior uveitis was found. Therefore dexamethasone eyedrops $0.1 \%$ and cyclopentolate eyedrops $1.0 \%$ were started, and over the next month the inflammation gradually settled and topical treatment was eventually stopped. However, within one week the patient attended again with a severe recurrence of the inflammation. Posterior synechiae and corneal oedema were seen, and once again a thickened posterior lens capsule was noted. A conjunctival swab at this stage showed no growth. Intensive topical steroids and cycloplegics was restarted, and during the next month 


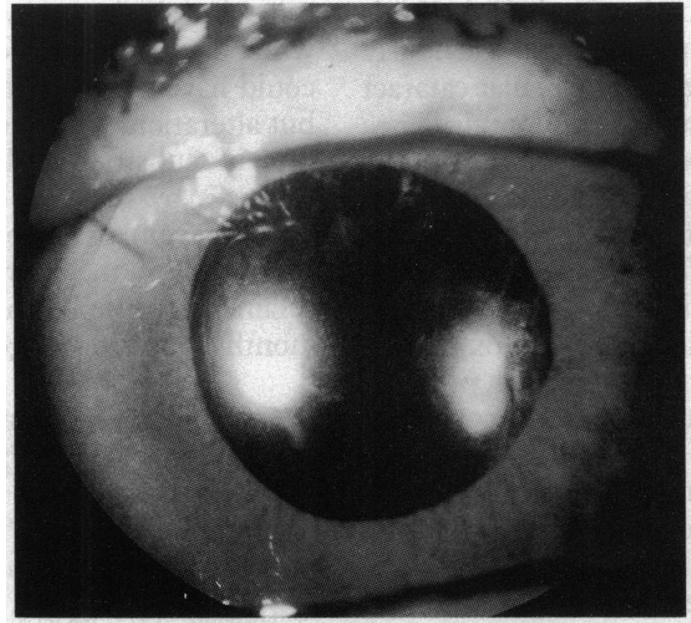

Figure 1 Abscess of the posterior lens capsule extending posteriorly into the vitreous.

the anterior chamber had settled sufficiently to allow a clear view of the lens capsule, which was seen to have developed a localised, whitish, dense opacity just inferotemporal to the visual axis. There was infiltrate extending back from this into the vitreous (Fig 1).

A diagnosis of endophthalmitis secondary to a posterior lens capsule abscess was made. Two days later a vitrectomy and capsulectomy were performed. An attempt was made to remove all abnormal looking capsule, leaving a peripheral annulus in which the lens was stable (Fig 2). During the procedure two specimens were taken: (1) capsular fluid, (2) vitreous. Intracameral antibiotics were not administered. A subconjunctival injection of cefuroxime $125 \mathrm{mg}$ was given, and postoperative treatment was cefuroxime eyedrops $50 \mathrm{mg} / \mathrm{ml}$ two hourly, dexamethasone eyedrops $0 \cdot 1 \%$ four times a day, and cyclopentolate eyedrops $1 \%$ four times a day. Oral flucloxacillin $250 \mathrm{mg}$ and pivampicillin $500 \mathrm{mg}$ four times a day and metronidazole 400 $\mathrm{mg}$ three times a day were given for seven days. All medication was tailed off over the next month. The eye has since remained quiet for six months and has a final visual acuity of $6 / 9$.

\section{BACTERIOLOGICAL FINDINGS}

Microscopy showed Gram-positive cocci and

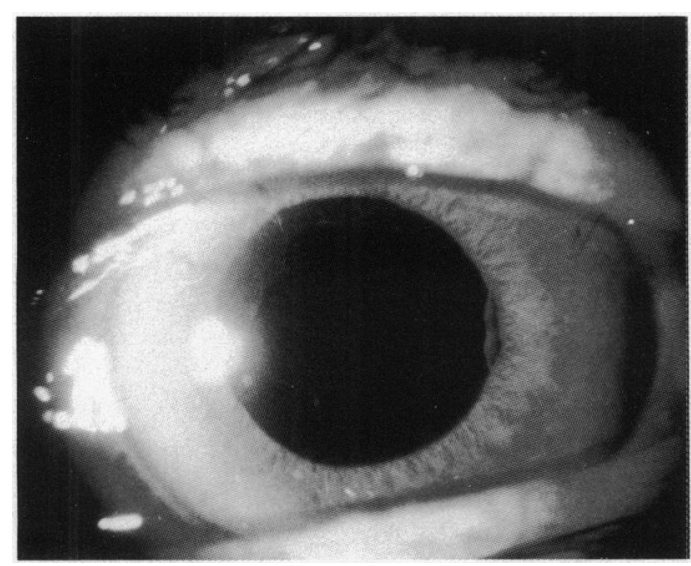

Figure 2 Postcapsulectomy and vitrectomy showing clearance of infective nidus and retention of intraocular lens. rods together with pus cells in the capsular fluid but no organisms in the vitreous.

Culture showed a mixed growth from both the capsular fluid and vitreous of Propionibacterium acnes and a single strain of coagulase negative Staphylococcus which showed colonial variation. The former was sensitive to penicillin and the latter to cloxacillin.

\section{Discussion}

The many reported cases of postoperative $P$ acnes endophthalmitis show a characteristic clinical course. The delay in onset is several months, and the inflammation then has a grumbling, insidious nature, often partially responding to antibiotics and steroids but recurring once topical treatment is stopped, as this case showed. ${ }^{6}$ The significance of the Staph epidermidis is difficult to assess. It is known to cause postoperative endophthalmitis, but a delay of five months before onset of symptoms is atypical.

The vector by which the organism gains entry to the eye is thought to be the intraocular lens. Vafidis $e t a l^{7}$ showed that, when sterile intraocular lenses were placed on the conjunctival flap during cataract surgery, $26 \%$ became contaminated with organisms, mainly Staph epidermidis. They suggested that minimising contact of the lens implant with the eye before insertion would reduce the chances of intraocular infection. Griffiths et $a l^{8}$ showed in vitro that Staph epidermidis can adhere to the plastic surface of the intraocular lens by producing a polysaccharide glycocalyx which acts like glue. Unfortunately this substance also seems to confer some resistance to antibiotics.

The intraocular lens used in this case was a one-piece, biconvex lens, posteriorly vaulted to an angle of $10^{\circ}$ with no laser ridge. Thus it is assumed that the posterior capsule was apposed to the back surface of the lens with no intervening space. The presence of an IOL has been shown to increase the intensity and duration of $P$ acnes infection in rabbit eyes, and possible explanations are that the IOL itself acts as a nidus for infection or that increased trauma during lens insertion may damage the bloodaqueous barrier, which will prolong the inflammatory response to intraocular bacteria.

The case presented here showed clearly that the continuing infective site was the posterior lens capsule. Exudative material on the capsule and IOL occurring in the active stage of an indolent, granulomatous iridocyclitis has been previously reported by Meisler et $a l^{10}$ Since the infection is thought to be associated with the lens capsule, it is more commonly found following extracapsular cataract extraction. Piest et al" examined capsule sacs that had been removed from cases of persistent, smouldering endophthalmitis and found pleomorphic bacilli in large numbers in the residual lens cortex and often in folds of capsule. In one case cultures were performed and $P$ acnes grown. They thought that the capsule acted as a protective barrier preventing a full blown disseminated endophthalmitis, which was reported following a Nd-YAG laser capsulotomy. Staph epidermidis 
has also been reported to cause endophthalmitis following Nd-YAG capsulotomy in a previously quiet eye five months after extracapsular cataract extraction. ${ }^{12}$ It was assumed that the low virulence micro-organisms had been sequestered in the capsular bag and released into the vitreous following laser treatment.

Ormerod et $a l^{13}$ have found experimentally in rabbits that the local environment in the vitreous just posterior to the lens has an oxidationreduction potential (Eh) and an oxygen pressure suitable for the growth of anaerobic organisms. They produced $P$ acnes endophthalmitis which was self-limiting. Many factors may have suppressed these infections including the rise in Eh with inflammation above the limiting value for the anaerobic bacteria. Administration of $100 \%$ oxygen to the anterior chamber has thus been put forward as a treatment for this condition.

Clinically, when topical treatment fails to control the inflammation, further intervention is required. Zambrano et al $^{14}$ have suggested that less severe cases can be treated with intravitreal vancomycin (with or without topical and intravenous antibiotics) and have shown successful results. They suggest vancomycin as the initial choice since it is effective against $P$ acnes and also coagulase negative staphylococci. Alternative antibiotics include methicillin and the cephalosporins. However, if the condition is initially severe or fails to respond to medical management, they recommend capsulectomy and pars plana vitrectomy together with intravitreal vancomycin. Our patient underwent such surgery without any antibiotics and the eye had improved dramatically within two days.

The intraocular lens alone does not appear to be the nidus of infection, since Meisler showed that whether or not the lens was removed in addition to the capsulectomy and vitrectomy made no difference to the successful outcome in their series of six cases. Driebe et $\mathrm{al}^{15}$ found only one out of 57 cases of pseudophakic endophthal- mitis in which the eye could not be sterilised while the intraocular lens was retained. Bacteria could have remained on the surface of the lens, but alterations in the local environment, such as loss of substrate cells and exposure to host defences by removal of the lens capsule, may have prevented regrowth. These theories are supported by the course in our patient who has retained her intraocular lens in a quiet eye for six months following surgery.

1 Perkins RE, Kundsin RB, Pratt MV, Abrahamsen I, Leibowitz HM. Bacteriology of normal and infected conjunctiva. $\mathcal{F}$ Clin Microbiol 1975; 1: 147-9.

2 Ormerod LD, Paton BG, Haaf S, Topping TM, Baker AS. Anaerobic bacterial endophthalmitis. Ophthalmology 1987; 94: 799-807.

3 Moore DC. Intralenticular abscess. Aust NZF Ophthalmo $1989 ; 17: 313-5$

4 Theodore FH. Bacterial endophthalmitis after cataract surgery. Int Ophthalmol Clin 1964; 4: 839-59.

5 Bode DB, Gelender H, Forster RK. A retrospective review of endophthalmitis due to coagulase-negative staphylococci. $\mathrm{Br}$ endophthalmitis due to coagula.

6 Jaffe GJ, Whitcher JP, Biswell R, Rodman Irvine A. Propionibacterium acnes endophthalmitis seven months after extracapsular cataract extraction and intraocular lens implantation. Ophthalmic Surg 1986; 17: 791-3.

7 Vafidis GC, Marsh RJ, Stacey AR. Bacterial contamination of intraocular lens surgery. Brf Ophthalmol 1984; 68: 520-3.

8 Griffiths PG, Elliot TSJ, McTaggart L. Adherence of Staphylococcus epidermidis to intraocular lenses. $\mathrm{Br} \mathcal{F}$ Ophthalmol 1989; 73: 402-6.

9 Nobe JR, Finegold SM, Rife LL, Edelstein MAC, Smith RE. Chronic anaerobic bacterial endophthalmitis in pseudophakic rabbit eyes. Invest Ophthalmol Vis Sci 1987; 28: 25963.

10 Meisler DM, Palestine AG, Vastine DW, Demartini DR, Murphy BF, Reinhart WJ, et al. Chronic Propionibacterium acnes endophthalmitis after extracapsular cataract extraction and intraocular lens implantation. Ophthalmic Surg 1986; 17: 791-3.

11 Piest KL, Apple DT, Kincaid MC, Roberts WA, Tetz MR, Price FW. Localised endophthalmitis: a newly described cause of the so-called toxic lens syndrome. $\mathcal{F}$ Cataract Refract Surg 1987; 13: 498-510.

12 Neuteboom GH, de Vries-Knoppert WA. Endophthalmitis after Nd-YAG laser capsulotomy. Doc Ophthalmol 1988; 70: 175-8.

13 Ormerod LD, Edelstein MAC, Schmidt GJ, Juarez RS, Finegold SM, Smith RE. The intraocular environment and experimental anaerobic bacterial endophthalmitis. Arch Ophthalmol 1987; 105: 1571-5.

14 Zambrano W, Flynn HW, Pflugfelder SC, Roussel TJ, Culbertson WW, Holland S, et al. Management options for Propionibacterium acnes endophthalmitis. Ophthalmolog 1989; 96: 1100-5.

15 Driebe WT, Mandelbaum S, Forster RK, Schwartz LK, Culbertson WW. Pseudophakic endophthalmitis. Ophthalmology 1986; 93: 442-7. 